\title{
Temporal integration differences between crossed and uncrossed stereoscopic mechanisms
}

\author{
ROBERT PATTERSON \\ Washington State University, Pullman, Washington \\ RON CAYKO, G. LARRY SHORT, RITA FLANAGAN, LINDA MOE, and ELOISE TAYLOR \\ Montana State University, Bozeman, Montana \\ and \\ PAM DAY \\ Washington State University, Pullman, Washington
}

\begin{abstract}
In this study, we investigated temporal integration of disparity information for crossed and uncrossed stereopsis. Across three experiments, exposure duration thresholds were measured for stereoscopic stimuli created from dynamic random-dot stereograms. In Experiment 1, an investigation of disparity detection showed that detection thresholds were equal for the crossed and uncrossed directions. In Experiment 2, an examination of duration limits for depth perception showed that critical durations were lower, and depth more veridical, for crossed depth than for uncrossed depth. In Experiment 3, an investigation of depth discrimination revealed that discrimination thresholds were lower for crossed depth than for uncrossed depth. These results suggest that crossed and uncrossed mechanisms differ in terms of their temporal integration properties at processing levels involving the computation and discrimination of depth.
\end{abstract}

In this study, we investigated the temporal integration of disparity information for crossed and uncrossed stereopsis. The motivation for this study came from previous research on stereoanomaly, which refers to an insensitivity to retinal disparity of a given direction, crossed or uncrossed, involving individuals with otherwise normal vision (Richards, 1970, 1971). In these original studies of stereoanomaly, Richards had observers discriminate the depth of stimuli presented with a large crossed or uncrossed disparity or with no disparity. One third of the observers were insensitive to depth in the crossed or uncrossed direction, but they could discriminate depth correctly in the other direction. The selective nature of stereoanomaly suggested that separate classes of neural mechanisms mediate crossed and uncrossed stereopsis, and that the stereoanomalous individuals possessed an impaired class of mechanism.

In Richards's $(1970,1971)$ studies, the test stimulus was exposed for $80 \mathrm{msec}$ in order to prevent vergence eye movements, which would alter the magnitude and possibly the direction of the disparity of the stimulus. Changes in disparity direction would allow a stereoanomalous observer to disguise a deficit. For example, an observer with crossed stereoanomaly could make convergence eye movements, thereby placing the stimulus in the uncrossed disparity region for which depth perception would be nor-

Correspondence concerning this article should be addressed to R. Patterson, Department of Psychology, Washington State University, Pullman, WA 99164-4820. mal. Brief exposures have also been used in other studies documenting large proportions of stereoanomalous observers (for review, see Mustillo, 1985).

It is likely that brief stimulus exposures may actually produce stereoanomaly. Patterson and Fox (1984) examined the performance of 98 observers on two depth perception tasks, one involving recognition of a briefly exposed stereoscopic Landolt $\mathrm{C}$ created from dynamic random-dot stereograms, and the other involving depth estimation of long-exposure stereoscopic afterimages (afterimages eliminated the confounding effects of vergence eye movements while providing long exposure durations). Patterson and Fox found that one third of the observers were stereoanomalous with the brief-exposure stereograms, but that almost all observers performed normally with the long-exposure afterimages.

These results suggested that stereoanomalous individuals do not have impaired stereoscopic mechanisms; rather, they simply possess a high duration threshold for disparity information. High duration thresholds could be produced by one or more factors, such as relatively low amplitude of the internalized disparity signal, high internal noise, sluggishness, sampling inefficiency, or under representation of the range of disparity to be processed. All of these factors could be compensated for by increasing the duration of stimulation. Such factors may vary among individuals such that disparity information accumulates at different rates, which would produce different disparity thresholds, with some individuals perceiving disparity quickly, others more slowly. Brief exposure could render 
disparity below duration threshold for the crossed or uncrossed direction, making performance "anomalous." A similar conclusion was reached by Finlay, Manning, Dunlop, and Dewis (1989), who also suggested that stereoanomaly may arise from the use of brief exposures rather than a "stereoscotoma."

Inspired by the possibility that an insensitivity to disparity information may be produced by brief stimulus exposure, we undertook an investigation to examine the temporal integration of disparity information for crossed and uncrossed stereopsis, looking for a difference between them (a signature of stereoanomaly). Because Richards's $(1970,1971)$ paradigm involved depth discrimination, we examined duration thresholds for the perception and discrimination of differing amounts of crossed or uncrossed depth. To provide comparison data, we also investigated duration thresholds for the detection of crossed or uncrossed disparity, on the notion that disparity detection reflects a different level of processing than does depth perception or depth discrimination, an idea considered later in the Discussion section. We wished to determine whether differences in temporal integration between crossed and uncrossed mechanisms are present throughout both detection and discrimination stages of binocular processing.

Three experiments were performed. In Experiment 1, duration thresholds for disparity detection were investigated; in Experiment 2, the duration at which depth becomes apparent in a stereoscopic stimulus was examined; and in Experiment 3, duration thresholds for depth discrimination were studied. To isolate the functioning of stereoscopic mechanisms, stimuli created from dynamic random-dot stereograms (Julesz, 1971) were employed in all experiments.

\section{GENERAL METHOD}

\section{Observers}

Sixty-nine male and female adults served as observers in one or more experiments, 3 of whom (R.C., R.F., E.T.) were co-authors of this paper. All observers had normal or corrected-to-normal acuity (tested by Orthorater, Bausch \& Lomb), and all were naive with respect to the purpose of the study at time of testing.

\footnotetext{
Apparatus

We employed a dynamic random-dot stereogram generation system described by Shetty, Brodersen, and Fox (1979) and Fox and Patterson (1981). The observer viewed a projection color TV system (Advent 1100; pixel size, 9'; stereogram luminance, $25 \mathrm{~cd} / \mathrm{m}^{2} ; 50 \%$ density; Michelson contrast between bright and dark dots of stereogram, 100\%) from a distance of $385 \mathrm{~cm}$ in Experiments 1 and 2, or a 19-in. Sharp color monitor (Model XM 1900; pixel size, 5.7'; stereogram luminance, $46 \mathrm{~cd} / \mathrm{m}^{2} ; 50 \%$ density; Michelson contrast between bright and dark dots of stereogram, 100\%) from a distance of $1.5 \mathrm{~m}$ in Experiment 3 .

The red and green guns of the projection TV or monitor were electronically controlled by a stereogram generator (hardwired device), which enabled red and green random-dot matrices to be displayed to the observer (approximately 5,000 dots in each matrix). Stereoscopic viewing was accomplished by placing red (Wratten 29) and green (Wratten 58) filters in front of the observer's eyes. The stereogram generator produced random dots and created disparity, which defined a stereoscopic stimulus (background dots correlated between eyes). All dots were replaced dynamically at a rate of $60 \mathrm{~Hz}$, which allowed the stimulus to be briefly exposed without monocu-
}

lar cues (Julesz \& Payne, 1968). The duration of the stimulus was controlled electronically in integer multiples of the frame duration of the display (16.7 $\mathrm{msec}$ ).

An optical programmer (moddified black and white video camera) transformed the two-dimensional achromatic stimuli that it scanned (e.g., a white square on a black background) into a stereoscopic stimulus on the TV or monitor. The voltage of the camera (whose scan rate was synchronized with that of the TV or monitor) was digitized and used as code to specify where disparity was inserted in the stereogram.

To promote stable binocular fixation, a small black dot was used for a fixation stimulus, located in the center of the display. Each observer was instructed to establish and maintain binocular fixation of the dot before initiating each trial.

\section{EXPERIMENT 1 Disparity Detection}

We first measured duration thresholds for detecting the presence of crossed or uncrossed disparity. Previous research on disparity detection has been equivocal. Uttal, Fitzgerald, and Eskin (1975) investigated disparity detection in random-dot stereograms and reported no difference in sensitivity between crossed and uncrossed directions. Manning, Finlay, Neill, and Frost (1987) also investigated disparity detection in random-dot stereograms and reported significant differences between the two directions (observers more sensitive to crossed than to uncrossed disparity).

\section{Method}

Four observers (R.C., R.F., E.T., R.S.) participated. On each trial, a briefly exposed, small stereoscopic square (size $=2^{\circ} \times 2^{\circ}$; area $=$ 4 square deg) was presented to either the left or the right of fixation (edge to edge separation between fixation dot and square $=1^{\circ}$ of arc). Employing a two-alternative forced-choice task, the observer verbally indicated the position of the square. Across trials, the duration of the square was varied among three or four values which corresponded to different levels of performance along a psychometric function; these values bracketed the duration corresponding to the $75 \%$ correct performance level. Across several sessions, 75 trials were collected at each duration ( 225 trials per condition). The $75 \%$ detection threshold was estimated by probit analysis of the percentage correct scores obtained at the different durations. Across disparity conditions, the square was presented at $18^{\prime}$ or $36^{\prime}$ of disparity in the crossed or uncrossed direction. In each session, the four disparity conditions were presented to the observer in random order.

\section{Results}

Interobserver differences were small, and the data were averaged across observers. Figure 1 shows the results for the four disparity conditions. Overall, thresholds were low and equal for the two disparity directions: average threshold was $45 \mathrm{msec}$ at $18^{\prime}$ of disparity, and it rose slightly to $50 \mathrm{msec}$ at $36^{\prime}$ of disparity.

These results are consistent with those of Uttal et al. (1975), who reported no differences in sensitivity between the two disparity directions. The results are inconsistent with those of Manning et al. (1987), who reported lower thresholds for crossed than for uncrossed disparity. However, the procedure used by Manning et al. for measuring thresholds was suspect: it involved a yes/no task and only a small number of trials, which renders the procedure open to criticism on the basis of response bias and unreli- 


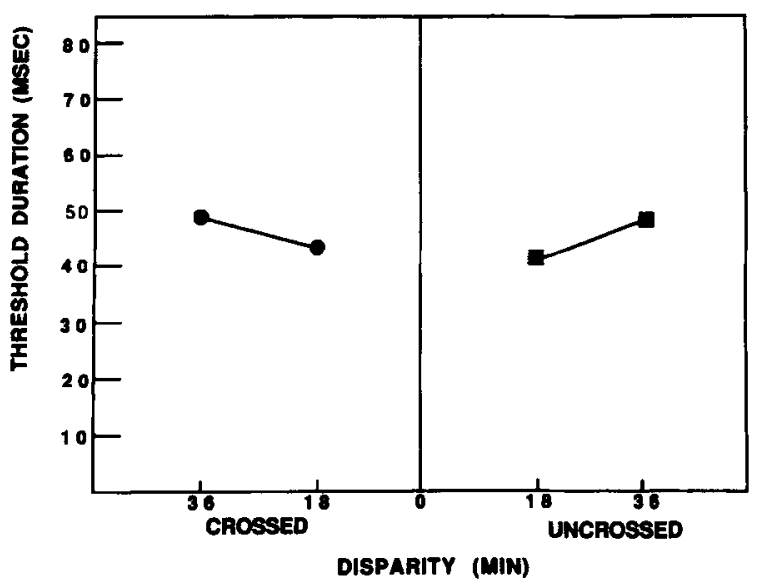

Figure 1. Duration thresholds for detection of $18^{\prime}$ and $36^{\prime}$ of disparity in crossed and uncrossed directions. Each data point is the mean of 4 observers. The largest standard error was $5.9 \mathrm{msec}\left(18^{\prime}\right.$ uncrossed disparity).

ability. Other differences between the Manning et al. (1987) study and the present investigation include presentation mode (haploscope vs. anaglyph) and stimulus disparity ( $15^{\prime}$ vs. $18^{\prime}$ and $36^{\prime}$ of arc), which also may have contributed to differences between the two sets of results.

We also measured duration thresholds for disparity recognition (i.e., cyclopean form recognition), a task similar to detection. Observers R.C. and R.F. participated. The stimulus was a stereoscopic Landolt $\mathrm{C}$ located in the center of the display; the $\mathrm{C}$ was presented with a disparity of $18^{\prime}$ or $36^{\prime}$ in either the crossed or the uncrossed direction. The outer diameter of the $\mathrm{C}$ was $4.5^{\circ}$, its width (horizontal cross section) was $18^{\prime}$, and its area was approximately 4.0 square deg. Over trials, gap position of the $\mathrm{C}$ was varied between the 3:00 and 9:00 positions. In a two-alternative, forced-choice task, observers verbally indicated gap position of the $\mathrm{C}$. The duration of the $\mathrm{C}$ was varied among three or four values, and recognition thresholds ( $75 \%$ correct performance) were estimated by probit analysis of the percentage correct scores ( 225 trials per condition).

Interobserver differences were again very small. Duration thresholds were low (below $90 \mathrm{msec}$ ), and there were no differences between crossed and uncrossed directions. Across disparity conditions, recognition thresholds were higher than detection by about $20 \mathrm{msec}^{1}$

The recognition task may also be viewed as a detection task. That is, an observer could have simply attended to the 3:00 or 9:00 position of the $C$ on each trial and looked for the presence or absence of a disparity step. Accordingly, the results of this experiment may be considered a replication of the results of the main detection experiment.

\section{EXPERIMENT 2 Critical Duration for Perceived Depth}

At detection threshold, the observers perceived the stereoscopic square without depth (it appeared in the plane of the display). To perceive depth, the exposure du- ration of the square had to be increased, indicating that the critical duration for perceiving depth is higher than that for detecting disparity. As the next step in our investigation, we measured the critical duration necessary for depth perception of the square under the same conditions as those in Experiment 1.

\section{Method}

Observers R.C., E.T., and R.S. participated. On each trial, the observer initiated exposure of the square; it was presented $1^{\circ}$ to the left or right of fixation. Because the task of estimating depth is subjective and sometimes difficult with brief durations, several exposures (typically 5-10) were used each trial, and depth was estimated according to two methods.

In the probe method, the observer indicated depth by instructing the experimenter to move and align a probe stimulus to appear to be coplanar with the perceived depth of the square. The probe was a vertically oriented steel rod located outside the stereoscopic display. Because the probe was an actual physical object, multiple cues to its depth position existed (e.g., disparity, size, interposition). It is likely that the observer made full use of these cues in judging depth of the probe with respect to that of the square. The lateral distance between probe and square was at least $1^{\circ}$ of arc, a distance large enough to preclude depth attraction or repulsion. The probe method was employed with crossed disparity only because we could not physically move the probe behind the display for uncrossed disparity. In the verbal method, the observer judged depth in terms of a percentage of the viewing distance to the screen. For example, if depth was equal to one half the distance, the observer responded, " 50 ."

Across trials, the stimulus was exposed to the observer, who made a judgment as to whether any depth was perceived (i.e., a yes/no task). The duration of the square was varied among four values which bracketed the transition phase from no depth perception to depth perception, corresponding to an interval of $66.8 \mathrm{msec}$ or less. As duration increased, the proportion of trials on which the observer perceived depth increased from $0 \%$ to $100 \%$. The critical duration for depth perception was defined as the lowest duration at which depth was perceived on $100 \%$ of the trials. Six trials ( 3 to the left and 3 to the right of fixation) were collected at each duration for each disparity, making a total of 24 trials per disparity condition.

This definition allowed for the possibility that some observers might perceive depth in the incorrect direction (e.g., front depth with uncrossed disparity) with brief exposure, an outcome of particular interest to us because we wished to determine the extent to which any anomalous depth perception would occur with brief durations. Such anomalous depth perception would not have been apparent had we defined the critical duration for depth perception as the lowest duration at which correct depth was perceived. In a sense, this experiment addressed two questions for each observer: (1) At what duration does depth first become apparent in our stimulus? (2) At that duration, is depth perception veridical?

\section{Results}

There were no differences in critical duration between left and right positions, so the data were averaged across the two positions. Verbal estimates (percentages) were converted to physical depth (in centimeters) by taking the product of the estimate and the viewing distance, and dividing by 100 , an approach which assumes that the observer correctly perceived his/her distance to the display screen. Verbal estimates defined in this way and probe measurements (crossed direction) were correlated +0.96 , indicating that the results were not peculiar to either measure of depth. The data reported below were obtained with the verbal method. 
Figure 2 shows critical duration for depth perception for the four disparity conditions. Critical duration for depth is higher than that for detection. For R.C. and E.T., there is an increase in critical duration with increases in disparity; the increase is slight in the crossed direction, and much greater in the uncrossed direction (the infinity symbol on the ordinate indicates depth perceived only with unlimited duration for E.T.). For R.S., the critical durations in the two directions are equal, but the depth with $36^{\prime}$ of uncrossed disparity is perceived in the wrong (crossed) direction - that is, reversed depth (open square). Richards (1971) also reported a stereoanomaly for which observers confused direction but not magnitude of depth with brief exposure. (No standard errors are depicted in Figure 2, because critical durations for depth for a given observer were the same value when retested, which is likely due to the coarse ordering of the duration scale - that is, steps of $16.7 \mathrm{msec}$ - causing duration estimates for each condition to be only one value.)

The magnitude of perceived depth at the critical duration was an increasing function of disparity magnitude with crossed disparity, while depth was weak (R.C., E.T.) or in the reversed direction (R.S.) with uncrossed disparity (Figure 3). We computed the magnitude of predicted depth on the basis of the geometry of stereograms ( $\mathrm{R}$. Cormack \& Fox, 1985): $d=(D \times S) /(I+S)$ for crossed disparity, and $d=(D \times S) /(I-S)$ for uncrossed disparity, where $d$ $=$ the predicted depth interval of the stimulus from the background, $D=$ the viewing distance, $S=$ the separation of half images, and $I=$ the interpupillary distance. The perceived depth with crossed disparity was close to the

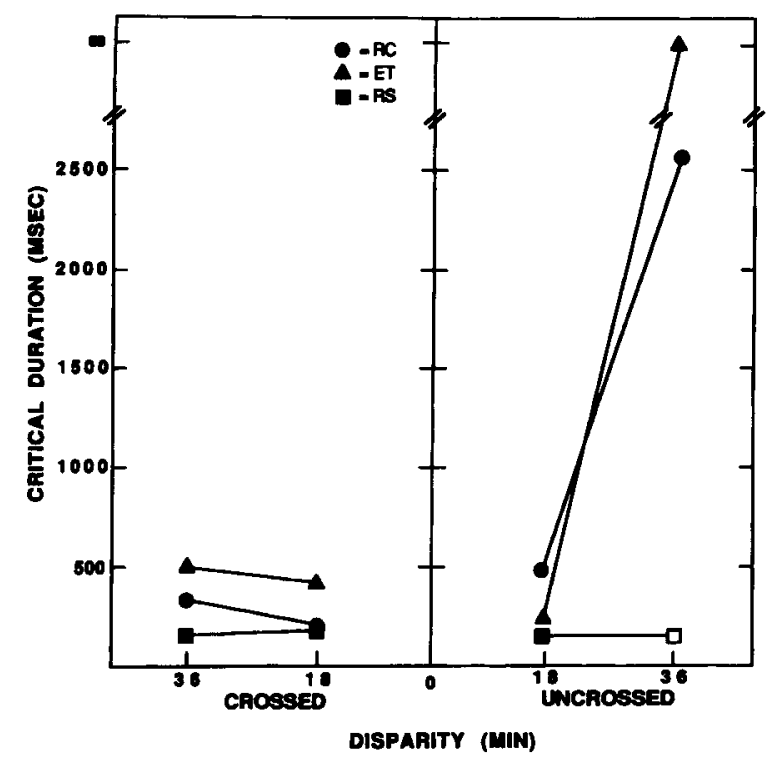

Figure 2. Critical duration for perceiving depth from $18^{\prime}$ and $36^{\prime}$ of disparity in crossed and uncrossed directions. Each data point represents critical duration for 1 observer. Infinity symbol on the ordinate indicates that depth was perceived only with unlimited duration for E.T. Open square indicates depth perceived with $3^{\prime}$ of uncrossed disparity was seen in the crossed direction for R.S. No standard errors are depicted, because critical durations were the same value when re-measured.

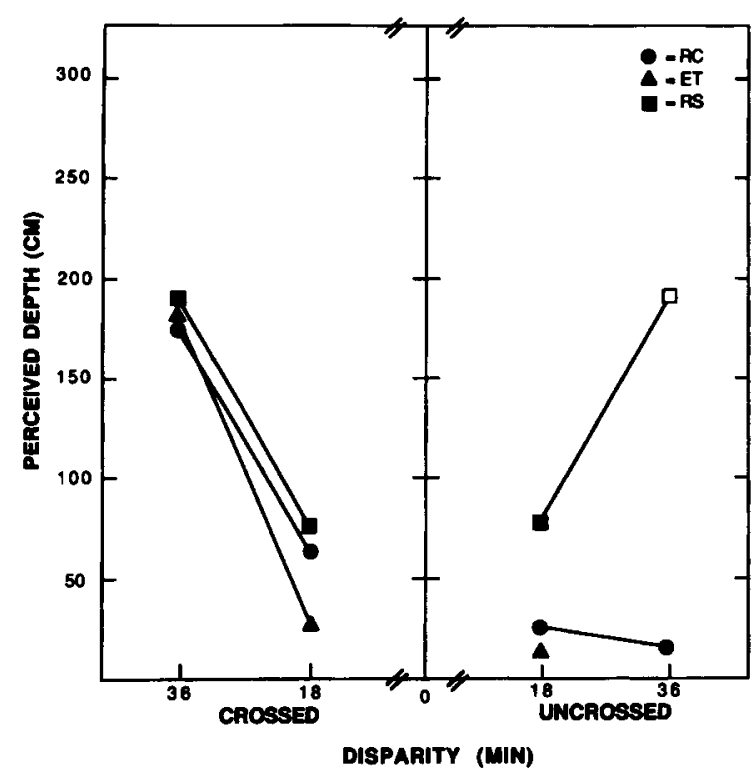

Figure 3. Perceived depth at critical duration for $18^{\prime}$ and $36^{\prime}$ of disparity in crossed and uncrossed directions. Each data point represents an average of six trials for 1 of 3 observers. Depth predicted on the basis of geometry for crossed disparity was $90 \mathrm{~cm}$ for $18^{\prime}, 146 \mathrm{~cm}$ for $36^{\prime}$; for uncrossed disparity, it was $171 \mathrm{~cm}$ for $18^{\prime}$, and $616 \mathrm{~cm}$ for $36^{\prime}$. Open square indicates that depth perceived with $36^{\prime}$ of uncrossed disparity was seen in the crossed direction for R.S. Missing symbol for E.T. at $36^{\prime}$ uncrossed indicates depth perceived only with unlimited duration (unmeasurable critical duration). The largest standard error was $24.9 \mathrm{~cm}$ (R.C., $18^{\prime}$ uncrossed disparity).

prediction ( $90 \mathrm{~cm}$ for $18^{\prime}, 146 \mathrm{~cm}$ for $36^{\prime}$ ), whereas the depth with uncrossed disparity was much less than the prediction $\left(171 \mathrm{~cm}\right.$ for $18^{\prime}, 616 \mathrm{~cm}$ for $\left.36^{\prime}\right)$. Patterson, Moe, and Hewitt (1992) have also reported that perceived depth is much less than prediction with uncrossed disparity, especially with brief exposures.

We also measured the critical duration for depth perception involving 48 other individuals. Methods similar to those used above were employed. This time a larger ( 9 square deg) stereoscopic square was used presented in the center of the display at a disparity of $36^{\prime}$. The larger stimulus was employed in an attempt to decrease durations near or below 180 msec, an estimate of the latency of vergence eye movements (Westheimer, 1954). We have observed in previous work that larger stimuli may produce a lower critical duration for depth perception (Patterson et al., 1992).

Of the 48 observers tested, 14 perceived depth correctly with crossed or uncrossed disparity; 22 perceived depth correctly with crossed disparity, but reversed depth with uncrossed disparity; 1 perceived depth correctly with uncrossed disparity, but reversed depth with crossed disparity; 1 reversed depth with crossed or uncrossed disparity; 4 perceived depth correctly with crossed disparity, but reported no depth perception with uncrossed disparity; and 6 showed no depth perception with either crossed or uncrossed disparity.

For observers with depth perception, most critical durations were under $100 \mathrm{msec}$ with crossed disparity, whereas 
they were higher with uncrossed disparity and depth was reversed in many cases (Figure 4). At the critical duration, the average perceived depth was close to prediction with crossed disparity, whereas the average depth fell short of prediction with uncrossed disparity (figure not shown).

Although some critical durations shown in Figure 4 were long enough to have permitted vergence eye movements, other durations were too brief to have done so. Of the 14 observers who perceived depth correctly with both crossed and uncrossed disparity, 6 had both critical durations at or below $180 \mathrm{msec}$. For these individuals, uncrossed durations were higher, on the average, than crossed durations by $66 \mathrm{msec}$. Differences in temporal integration for depth perception exist between the crossed and uncrossed directions under conditions that would preclude vergence eye movements.

The results of Experiment 2 show that perceived depth with crossed disparity is more sensitive and accurate than depth with uncrossed disparity. Critical durations for depth perception are lower and fewer depth reversals occur with crossed disparity.

Although the results of Experiment 2 reveal differences between crossed and uncrossed stereopsis, the measurement of perceived depth was subjective, which renders the method open to criticism on the basis of differing re-

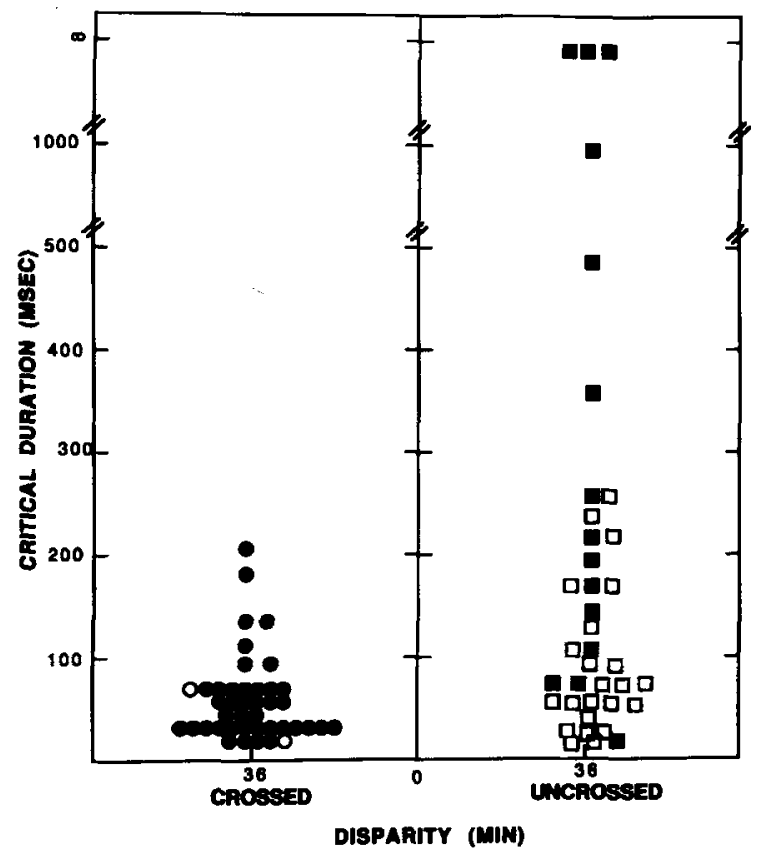

Figure 4. Critical duration for perceiving depth from $36^{\prime}$ of disparity in crossed and uncrossed directions. Each data point represents critical duration for 1 observer. Four missing symbols for uncrossed disparity indicate no uncrossed depth perception for those individuals. Infinity symbol on the ordinate indicates that depth was perceived only with unlimited duration. Open circles indicate that depth perceived with crossed disparity was seen in the uncrossed direction, and open squares indicate that depth perceived with uncrossed disparity was seen in the crossed direction. No standard errors are depicted, because critical durations were the same value when re-measured. sponse criteria or bias. Experiment 3 was performed to remedy this problem.

\section{EXPERIMENT 3 Depth Discrimination}

In this experiment, we wished to investigate the critical duration for depth perception in a forced-choice objective task. To do so, we measured duration thresholds for the discrimination of depth differences between crossed or uncrossed stimuli.

\section{Method}

This experiment was performed in two phases. In the first phase, 2 experienced psychophysical observers served. On each trial, two stereoscopic squares (size $=2.0$ square $\mathrm{deg}$ ) were presented side by side, both of them with either crossed or uncrossed disparity, for a duration of $66 \mathrm{msec}$. One square, the standard, was presented at a fixed disparity of $28.6^{\prime}$; the other square, the comparison, was presented at a disparity of $22.8^{\prime}$ or $34.4^{\prime}$, either slightly in front of or behind the standard. The observers's task was to diseriminate the depth position of the comparison in relation to that of the standard. One hundred trials were collected for the crossed and uncrossed directions for each observer.

In the second phase, 15 observers served. On each trial, two stereoscopic squares (size $=1.0$ or 1.15 square deg) were again exposed side by side, both of them with either crossed or uncrossed disparity. The standard square was presented at a fixed disparity of $17.1^{\prime}$; the comparison square was presented at a disparity of $11.4^{\prime}$ or $22.8^{\prime}$, slightly behind or in front of the standard. As in Phase 1, the observer's task was to discriminate the depth position of the comparison and standard. Over trials, duration of the two squares was staircased according to a 3-1 rule. For every three correct responses in a row, the duration of the squares was decreased one step, whereas for every single incorrect response, the duration was increased. Step size was $16.7 \mathrm{msec}$, one frame of the display. This staircase rule converges upon the $79 \%$ performance level (Wetherill \& Levitt, 1965). Each staircase entailed about 60-70 trials; the last 6 turnarounds were averaged together to provide an estimate of threshold.

Of the 15 observers, 8 performed four staircases (four thresholds) for each crossed and uncrossed direction with the 1.0 square deg stimuli; the other 7 observers performed two staircases (two thresholds) for each crossed and uncrossed direction with the 1.15 square deg stimuli. The thresholds were averaged together under each condition for each observer to provide a mean threshold. Each observer participated in one practice session before formal data collection, which involved two subsequent sessions. At the beginning of these sessions, the observers were tested on whether they could perform the discrimination task correctly ( $100 \%$ correct performance) with the stimuli exposed for an unlimited duration, and all but 1 observer could do so for both crossed and uncrossed directions (the remaining observer could do so for the crossed but not the uncrossed direction).

For half the staircases, the standard square was positioned to the right of fixation and the comparison square to the left; for the other staircases, the opposite was true. Small differences in the apparent size or lateral position of the squares produced by differences in their perceived depth were controlled for by introducing slight random changes in size and position of one or the other square on each trial.

We selected our stimulus parameters so that trial durations would typically fall at or below $167 \mathrm{msec}$, which ensured that the trials would be performed without vergence eye movements (which would change horopter position and possibly confound crossed and uncrossed disparity). However, in several cases the observer performed unsuccessfully with the 167 -msec trial duration; he/she had runs of trials with many errors and failed to achieve three correct responses 
in a row. These cases included 6 observers with uncrossed disparity and 2 observers with crossed disparity, all involving the 1.0 square deg stimuli, and 2 observers with uncrossed disparity involving the 1.15 square deg stimuli. According to the staircase rule, we should have increased trial duration for each incorrect response, but instead we maintained duration at $167 \mathrm{msec}$ during these runs to prevent vergence eye movements, enabling us to assign with certainty a given performance to the crossed or uncrossed direction. For these cases, thresholds were greater than $167 \mathrm{msec}$ by some unknown amount (as is indicated in Figure 5). In several other cases, the observer had runs of trials with many correct responses with the $16.7-\mathrm{msec}$ trial duration. These cases included 1 observer with the 1.0 square deg stimuli and 2 observers with the 1.15 square deg stimuli, all involving crossed disparity. Because $16.7 \mathrm{msec}$ represented the shortest duration that we could employ (one frame of our display), we could not decrease the trial duration below that value. In these cases, the thresholds were less than $16.7 \mathrm{msec}$ by some unknown amount (Figure 5).

\section{Results}

In the first phase, involving the 2 observers, we found that depth of standard and comparison was discriminated correctly on nearly $100 \%$ of the trials (J.M., $98 \%$; K.P., $99 \%$ ) when the stimuli were presented with crossed disparity, but on only about $50 \%$ of the trials (J.M., $50 \%$; K.P., $54 \%$ ) when they were presented with uncrossed disparity (figure not shown). Apparently, a duration of $66 \mathrm{msec}$ was above threshold for crossed disparity but below threshold for uncrossed disparity for these observers.

With respect to the second phase, involving the 15 observers, Figure 5 shows the duration thresholds for depth discrimination for the crossed and uncrossed directions. The filled symbols indicate discrimination measured with the 1.0 square deg stimuli; the open symbols represent discrimination measured with the 1.15 square deg stimuli. The thresholds for discrimination of crossed depth were typically lower than the thresholds for discrimination of un-

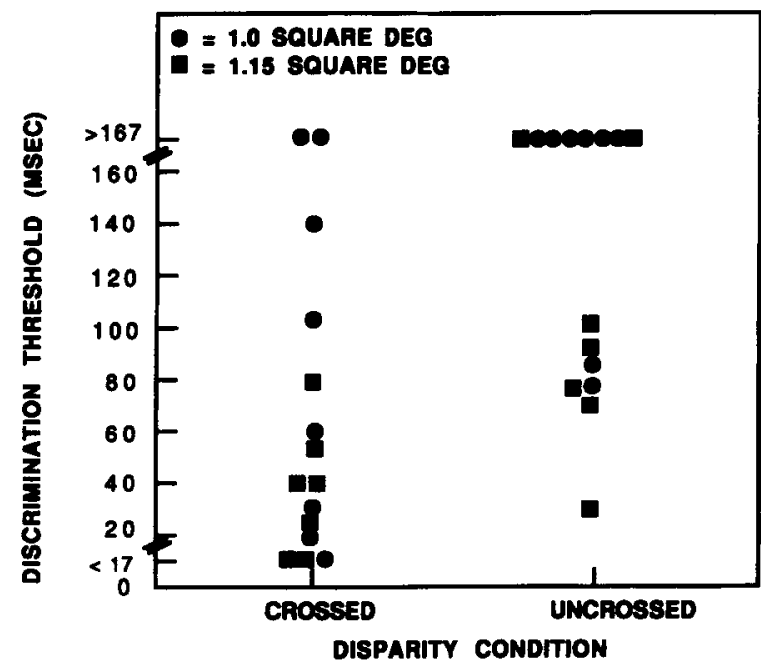

Figure 5. Duration thresholds for depth discrimination in crossed and uncrossed directions. Each data point represents threshold for 1 observer. Filled circles indicate thresholds obtained with the 1.0-square-deg stimuli, and open circles indicate thresholds obtained with the 1.15-square-deg stimuli. See text for details. crossed depth. With the smaller stimulus, the median threshold for crossed depth was $87 \mathrm{msec}$, whereas it was greater than $167 \mathrm{msec}$ for uncrossed depth. For 6 of the 8 observers tested with the smaller stimulus, ratios of uncrossed threshold to crossed threshold were all greater than 1.0 , with most ratios in the $2-3$ range (for the 2 remaining observers, the ratios were undefined because both uncrossed and crossed thresholds were greater than 167 msec). With the larger stimulus, the median threshold for crossed depth was $45 \mathrm{msec}$, whereas it was $94 \mathrm{msec}$ for uncrossed depth. For the 7 observers tested with the larger stimulus, the ratios of uncrossed threshold to crossed threshold were all greater than 1.0, with most ratios again in the 2-3 range. Each observer reported that he/she could make successful discriminations with long durations because the depth of the stimuli could be perceived, whereas he/she could not do so with brief durations because the depth could not be perceived.

The results of Experiment 3 show that the discrimination of crossed depth occurs at shorter durations than does the discrimination of uncrossed depth.

\section{DISCUSSION}

This study shows that crossed and uncrossed stereopsis differ in terms of their temporal integration properties at processing levels involving the computation and discrimination of depth. For most but not all observers, integration occurs over shorter time intervals, and depth is more veridical, for crossed than for uncrossed stereopsis. Such differences are not revealed for disparity detection.

We interpret the results of Experiment 3 as reflecting depth discrimination, not disparity discrimination, for the following reason. Our observers were told to judge whether the comparison stimulus appeared in front or back of the standard. Although front depth would indicate greater disparity and back depth less disparity for crossed stimuli, the opposite would be true for uncrossed stimuli. For observers to discriminate the depth relations between stimuli in both crossed and uncrossed directions successfully, responses would need to be based on actual depth. Our observers stated that their ability to perform the discrimination task successfully was based on the perceived depth of the stimuli. This interpretation of Experiment 3 is consistent with the results of Experiment 2, in which there were differences in depth perception between crossed and uncrossed directions. (The same logic would apply in ruling out decorrelation detection as a basis of performance in Experiment 3.)

In making a distinction between depth and disparity, note that disparity by itself is an ambiguous depth cue. In real-world viewing, variation in viewing distance between an observer and stimuli produces changes in disparity magnitude, even though the depth may remain constant among objects. For veridical depth perception to occur, the visual system perceptually rescales disparity for different viewing distances, a process called depth constancy (Ono \& Comerford, 1977; Wallach \& Zuckerman, 1963). We suggest that differences in temporal integration be- 
tween crossed and uncrossed stereopsis may be related to depth constancy. At brief durations, many observers are unable to perceive the correct sign or magnitude of depth in the crossed or uncrossed direction.

This may provide an explanation for stereoanomaly. Stereoanomaly may arise from the use of brief exposure (Finlay et al., 1989; Patterson \& Fox, 1984; see also Manning, Finlay, Dewis, \& Dunlop, 1992), which renders stereoscopic stimuli below duration threshold for the crossed or uncrossed direction. We predict that changes in exposure duration should influence the proportion of individuals classified as stereoanomalous. Very brief durations (e.g., $30 \mathrm{msec}$ ) should produce a large proportion of stereoanomalous observers, because stimulation would be below duration threshold for many of them, whereas long durations (e.g., $180 \mathrm{msec}$ ) should produce a smaller proportion of stereoanomalous observers, because stimulation would exceed many thresholds. This would imply that "stereoanomaly" is distributed continuously throughout the population, representing a normal state of functioning.

Our results in turn provide a challenge to the notion of discrete classes of stereoscopic mechanisms. In the original statement of this idea, Richards $(1970,1971)$ claimed that stereoanomalous individuals possess a selective impairment that is indicative of separate classes of neural mechanisms for crossed and uncrossed stereopsis. However, our results, suggesting that stereoanomaly may arise from the use of brief stimulus exposures, undercut the notion of neural impairment and thus the notion of discrete classes of mechanism. At the neurophysiological level, Poggio and colleagues (e.g., Poggio \& Fischer, 1977; Poggio, Motter, Squatrito, \& Trotter, 1985) have provided evidence for discrete categories of disparity-selective cells in the cortex of cats and monkeys, but LeVay and Voigt (1988) have recently shown that the response types of disparity-tuned cells in the cat belong to a continuum, not to discrete categories (see also Freeman \& Ohzawa, 1990). Thus too, L. K. Cormack, Stevenson, and Schor (1993) have shown that disparity tuning functions measured psychophysically exhibit inhibition between disparities of the same or different sign, providing evidence for a continuum of disparity-tuned mechanisms. Note that no difference between crossed and uncrossed disparity was observed in the L. K. Cormack et al. study, which involved interocular correlation detection, nor in the structure of the disparity channels implied by their masking data. On the basis of the results of the present investigation, no difference would be expected, because the task employed by L. K. Cormack et al. (1993) did not rely on depth perception or on depth discrimination.

\section{REFERENCES}

Cormack, L. K., Stevenson, S. B., \& Schor, C. M. (1993). Disparitytuned channels of the human visual system. Visual Neuroscience, 10, 585-596.

Cormack, R. [H.], \& Fox, R. (1985). The computation of disparity and depth in stereograms. Perception \& Psychophysics, 38, 375-380.

Finlay, D. C., Manning, M. L., Dunlop, D. P., \& Dewis, S. A. (1989). Difficulties in the definition of "stereoscotoma" using temporal detection thresholds of dynamic random-dot stereograms. Documenta Ophthalmologica, 72, 161-173.
Fox, R., \& Patterson, R. (1981). Depth separation and lateral interference. Perception \& Psychophysics, 30, 513-520.

Freeman, R. D., \& OhzaWA, I. (1990). On the neurophysiological organization of binocular vision. Vision Research, 30, 1661-1676.

JuLESZ, B. (1971). Foundations of cyclopean perception. Chicago: University of Chicago Press.

Julesz, B., \& Payne, R. (1968). Difference between monocular and binocular stroboscopic movement perception. Vision Research, 8 , 433-444.

LEVAY, S., \& VoIGT, T. (1988). Ocular dominance and disparity coding in cat visual cortex. Visual Neuroscience, 1, 395-414.

Manning, M. L., Finlay, D. C., Dewis, S. A., \& Dunlop, D. B. (1992). Detection duration thresholds and evoked potential measures of stereosensitivity. Documenta Ophthalmologica, 79, 161-175.

Manning, M. L., Finlay, D. C., Neill, R. A., \& Frost, B. G. (1987). Detection threshold differences to crossed and uncrossed disparities. Vision Research, 27, 1683

Mustillo, P. (1985). Binocular mechanisms mediating crossed and uncrossed stereopsis. Psychological Bulletin, 97, 187.

ONo, H., \& COMERFord, T. (1977). Stereoscopic depth constancy. In W. Epstein (Ed.), Stability and constancy in visual perception: Mechanisms and processes (pp. 91-128). New York: Wiley.

Patterson, R., \& Fox, R. (1984). The effect of testing method on stereoanomaly. Vision Research, 25, 403-408.

Patterson, R., Moe, L., \& Hewitt, T. (1992). Factors that affect depth perception in stereoscopic displays. Human Factors, 6, 655-667.

Poggio, G. F., \& Fischer, B. (1977). Binocular interaction and depth sensitivity of striate and prestriate cortical neurons of the behaving rhesus monkey. Journal of Neurophysiology, 40, 1392.

Poggio, G. F., Motter, B. C., SQuatrito, S., \& Trotter, Y. (1985). Responses of neurons in visual cortex (V1 and V2) of the alert macaque to dynamic random-dot stereograms. Vision Research, 25, 397.

RICHARDS, W. (1970). Stereopsis and stereoblindness. Experimental Brain Research, 10, 380.

RICHARDS, W. (1971). Anomalous stereoscopic depth perception. Journal of the Optical Society of America, 61, 410-414.

ShetTy, S. S., Brodersen, A. J., \& Fox, R. (1979). System for generating dynamic random-element stereograms. Behavior Research Methods \& Instrumentation, 11, 485-490.

UtTal, W. R., Fitzgerald, J., \& Eskin, T. E. (1975). Parameters of tachistoscopic stereopsis. Vision Research, 15, 705-712.

WALLACH, H., \& ZUCKERMAN, C. (1963). The constancy of stereoscopic depth. American Journal of Psychology, 76, 404-412.

WESTHEIMER, G. (1954). Eye movement responses to a horizontally moving visual stimulus. American Medical Association Archives of Ophthalmology, 52, 932-941.

WeTherILL, G. B., \& LeVITT, H. (1965). Sequential estimation of points on a psychometric function. British Journal of Mathematical \& Statistical Psychology, 18, 1-10.

\section{NOTE}

1. With random-dot stereograms, decorrelation information is necessarily introduced to corresponding retinal areas when the subset of dots in one eye's view is shifted to create disparity. The stimuli in Experiment 1 may have represented areas of interocular decorrelation (disparity-shifted area) flanked by regions of +1.0 interocular correlation; the observers may have spatially localized decorrelation rather than disparity, a problem that also arises in other investigations of disparity detection (e.g., Manning et al., 1987). However, a decorrelation explanation of detection is unlikely: increasing disparity increases the size of the area of decorrelation. Assuming that increasing size of decorrelation should lower thresholds via spatial integration, increasing disparity should have lowered thresholds if performance was based on decorrelation. However, increasing disparity increased thresholds slightly, suggesting that detection performance was not based on decorrelation. The results of Experiment 3 cannot be explained by decorrelation detection.

(Manuscript received May 3, 1994 revision accepted for publication February 23, 1995.) 